\title{
Understanding deposition rate loss in high power impulse magnetron sputtering: $I$. Ionization-driven electric fields
}

\author{
N Brenning, C Huo, Daniel Lundin, M A Raadu, C Vitelaru, G D Stancu, \\ T Minea and Ulf Helmersson
}

\section{Linköping University Post Print}

N.B.: When citing this work, cite the original article.

Original Publication:

N Brenning, C Huo, Daniel Lundin, M A Raadu, C Vitelaru, G D Stancu, T Minea and Ulf Helmersson, Understanding deposition rate loss in high power impulse magnetron sputtering: I. Ionization-driven electric fields, 2012, Plasma sources science \&amp; technology (Print), (21), 2, 025005.

http://dx.doi.org/10.1088/0963-0252/21/2/025005

Copyright: Institute of Physics

http://www.iop.org/

Postprint available at: Linköping University Electronic Press http://urn.kb.se/resolve?urn=urn:nbn:se:liu:diva-77328 


\title{
Understanding deposition rate loss in high power impulse magnetron sputtering
}

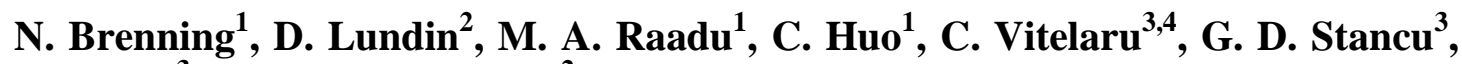 \\ T. Minea ${ }^{3}$, and U. Helmersson ${ }^{2}$
}

\author{
1 Division of Space and Plasma Physics, School of Electrical Engineering, Royal Institute of \\ Technology, SE-100 44 Stockholm, Sweden \\ 2 Plasma \& Coatings Physics Division, IFM-Materials Physics, Linköping University, SE-581 83 \\ Linköping, Sweden \\ ${ }^{3}$ Laboratoire de Physique des Gaz et Plasmas, UMR 8578 CNRS, Université Paris Sud-XI, 91405 \\ Orsay Cedex, France \\ ${ }^{4}$ Physics Department, Faculty of Physics Al I Cuza University, Bd. Carol No 11, Iasi, 700506,
Romania
}

E-mail: nils.brenning@ee.kth.se

\begin{abstract}
The lower deposition rate for high power impulse magnetron sputtering (HiPIMS) compared to direct current magnetron sputtering for the same average power is often reported as a drawback. The often invoked reason is back-attraction of ionized sputtered material to the target, due to a substantial negative potential profile from the location of ionization towards the cathode. Emitting and swept Langmuir probes have yielded space- and time resolved electric potential profiles and electron energy distributions, Rogowski coils have been used to obtain current density distributions. Also, space- and time resolved, and fast imaging techniques show how the time evolution of the discharge structure varies with gas pressure and species. This combined data set is here used to benchmark two different types of plasma models for different regions of the HiPIMS discharges, with special focus on the problem of electric fields $E_{z}$ in the high density plasma region and their effect on the transport of ionized sputtered material. We propose two different mechanisms to be dominating in different regions: "ionization driven" $E_{z, \text { ioniz }}$ in a rather stable ionization region extending a few $\mathrm{cm}$ from the target, and "transport driven" $E_{z, \text { trans }}$ in the highly dynamic surrounding bulk plasma.
\end{abstract}




\section{Introduction}

One of the main advantages with high power impulse magnetron sputtering (HiPIMS) is the dramatic increase of the degree of ionization of the metallic vapor [1,2] which opens a completely new perspective in the engineering and design of new thin film materials [1]. However, a problem with using ions is that the electric potential applied to the target and the resulting electrical field $E_{z}$ can reduce the deposition rate seriously if they extend outside the cathode sheath, into the dense plasma. In Christietype models (see Figure 1a) $[3,4]$ a key variable is the back-attraction parameter, $\beta$, of ionized metal $\mathrm{M}^{+}$. An electric field $E_{z}$ in the plasma (see Figure $1 \mathrm{~b}$ ) can turn such ions around, increase $\beta$, and decrease the deposited fraction $\delta$ of the $\mathrm{M}^{+}$ions. Recent probe data $[5,6]$ have shown that in HiPIMS there can be a potential uphill from the sheath edge to the bulk of the plasma that can vary at least in the range 7-60 V, with stronger $E_{z}$ generally observed closer to the target, as well as for stronger magnetic field, for stronger applied power, and during the early stage of the HiPIMS pulse.

For a discussion on $E_{z}$ in HiPIMS discharges we divide the plasma into two regions (see Figure 1c) in which different mechanisms for $E_{z}$ are proposed to dominate. In the ionization region (IR) extending a few $\mathrm{cm}$ from the target there are “ionization driven" $E_{z, \text { ioniz }}$, essentially forced by the energy and particle balance conditions for a self-sustained discharge. In the surrounding bulk plasma (BP) "transport driven" fields $E_{z, \text { trans }}$ are needed to close the discharge current $I_{D}$ by electron transport across the magnetic field lines to the anode. 
a)

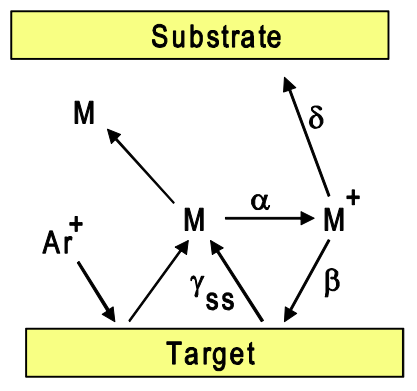

b)

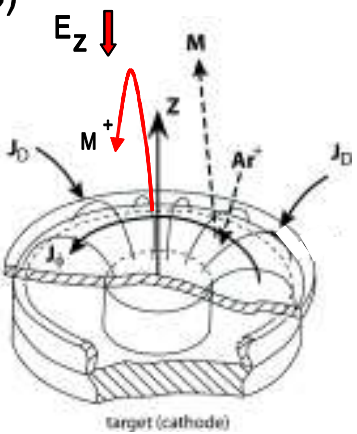

c)

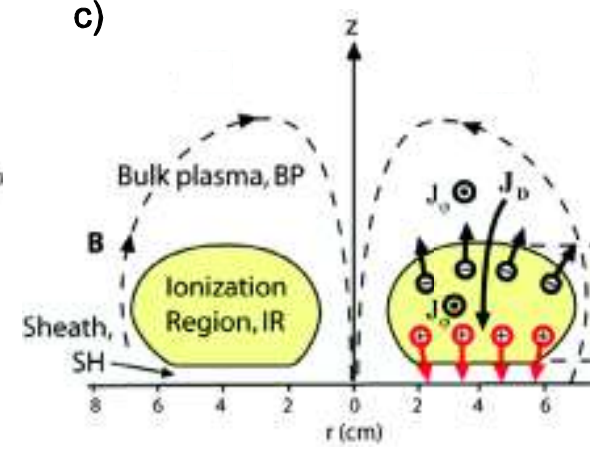

Figure 1. a), b) The role of $E_{z}$ for the $M^{+}$particle dynamics in magnetron sputtering. c) Crosssectional view of the magnetron describing two plasma regions: the ionization region (IR) and the bulk plasma (BP) region, where different mechanisms are proposed to give rise to $E_{z}$.

\section{Ionization driven electric fields $E_{z, \text { ioniz }}$}

As a basic driving mechanism for generation of $E_{z}$ fields in the ionization region it is here proposed the need to heat the electrons enough for a self sustained ion particle balance as shown in Figure 2. We first pose the question: assuming that there are no electric fields outside the plasma sheath region, under what conditions can the ion losses from the ionization region be replaced by electron impact ionization? For parameters where they cannot, we will have to return to the much more difficult question of how $E_{z}$ in the ionization region can help to alleviate the situation. We will refer to such fields as "ionization driven" and denote them by $E_{z, \text { ioniz }}$.

Consider in Figure 2 an $\mathrm{Ar}^{+}$ion that hits the target and emits a secondary electron with a probability $\gamma_{s e} \approx 0.1$. For steady-state this has to, on average, set a chain of events into motion that leads to another $\mathrm{Ar}^{+}$ion hitting the target. This condition is quantified as follows. Under the assumption $E_{z, \text { ioniz }}=0, U_{S H}=U_{D}$, where $U_{S H}$ and $U_{D}$ are the sheath and discharge potentials, respectively, it is found that the emitted electron is accelerated to the energy $e U_{D}$ across the sheath. With an effective 
cost of ionization $e U_{i, E F F}$, and a fraction $\beta_{A r^{+}}$of the $\mathrm{Ar}^{+}$ions drawn to the target, we can define a Townsend product as

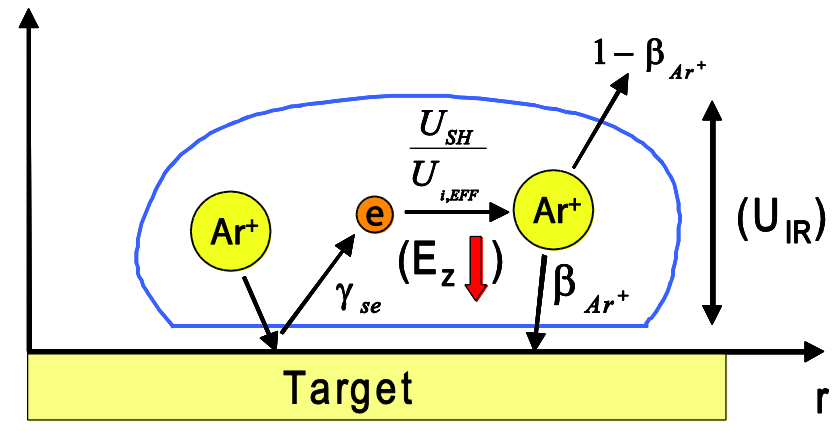

Figure 2. Model of the ionization region showing the reactions involved in $\mathrm{Ar}^{+}$ion particle balance. The parentheses around $E_{z}$ and the potential difference across the ionization region, $U_{\mathrm{IR}}$, indicate that these quantities are assumed to be zero in Eq. 1 and in the runs of the ionization region model (IRM I).

$$
\Pi_{\text {Townsend }}=\gamma_{\text {se }} \frac{U_{D}}{U_{i, E F F}} \beta_{A r^{+}} .
$$

In steady-state, $\Pi_{\text {Townsend }}=1$, while $\Pi_{\text {Townsend }}<1$ corresponds to a discharge that will be depleted of plasma and become extinguished. $\Pi_{\text {Townsend }}>1$ can be seen as a breakdown condition: a discharge can ignite but will not be in steady-state. As a numerical example we take the parameters $\gamma_{s e}=0.1, U_{D}=600 \mathrm{~V}, U_{i, E F F}=35 \mathrm{eV}^{*}$ (see also reference 7), and a rather efficient collection of ions to the target, $\beta_{A r^{+}}=0.8$. This gives $\Pi_{\text {Townsend }}=1.37$, i.e. the breakdown condition is satisfied. As this numerical example demonstrates, it is in principle possible to have a discharge without ionization driven $E_{z, \text { ioniz }}$ fields, but the physical picture is far too simplistic to prove certain that there is a large enough margin above the steady-state value. Better modeling is therefore needed including a physically motivated estimate of $\beta_{A r^{+}}$,

\footnotetext{
* The effective costs of ionization $U_{i, E F F}$ used in this paper are estimated from three contributing mechanisms, (1) the collisional energy loss including ionization which depends on electron energy [7], (2) heating the new electron to the bulk electron temperature, and (3) the energy cost of electron flow from the ionization region to the bulk plasma.
} 
realistic cross-sections for all species present, density growth and decay during a HiPIMS pulse, Penning ionization, time-varying $U_{D}(t)$, gas rarefaction, the transition to self-sputtering, etc.

In order to more carefully study if a discharge can be sustained assuming $E_{z, \text { ioniz }}=0$ simulations of the ionization region have been run using IRM 1 , which is a global plasma model $[8,9]$ that includes these effects and can flexibly be adjusted to different discharges. As a model system, data has been taken from a "reference discharge" in Ar with a $15 \mathrm{~cm}$ in diameter Al target mounted on a slightly unbalanced magnetron. The discharge is driven by a Sinex I HiPIMS power supply from Chemfilt Ionsputtering, delivering $100 \mu$ s long discharge pulses with a peak current of $105 \mathrm{~A}$ and a peak voltage of $700 \mathrm{~V}$, which decreases monotonically to $200 \mathrm{~V}$ as the pulse decays. Typical $I_{D}(t)-U_{D}(t)$ characteristics can be found in works by Bohlmark et al. [10]. The reason for this choice is that this particular magnetron has been extensively diagnosed $[2,10,11,12,13]$.

In IRM I, $I_{D}(t)$ and $U_{D}(t)$ are input parameters. One key output parameter is the "required power to the electrons" in form of the fraction $F_{P W R}$ of the electric input power $I_{D} U_{D}(t) . \quad F_{P W R}$ is normally adjusted using a self-consistency condition $I_{i}\left(1+\gamma_{s e}\right)=I_{D}(t)$, where $I_{i}\left(1+\gamma_{s e}\right)$ is the calculated sum of the ion current and secondary electron current to the target. The secondary emission coefficient, $\gamma_{s e}$ varies in time since it depends on the time-varying fraction of $\mathrm{Ar}^{+}$to $\mathrm{M}^{+}$ions $\left(\gamma_{s e} \approx 0.1\right.$ for $\mathrm{Ar}^{+}$while $\gamma_{\text {se }} \approx 0$ for $\mathrm{M}^{+}$).

In this work, the model is not run for these self consistent solutions, since the artificial condition $E_{z, \text { ioniz }}=0$ has been imposed. When this is the case, the only source of energy in the plasma are secondary emitted electrons accelerated across the sheath, and $F_{P W R}$ is locked to the value $F_{P W R}=\gamma_{s e} /\left(1+\gamma_{s e}\right)$. An advantage with the 
assumption $E_{z, \text { ioniz }}=0$ is that the fraction to the target $\beta_{A r^{+}}$can be modeled rather realistically. No ion flux in the ionization region is electric field driven, and $\beta_{A r^{+}}$is
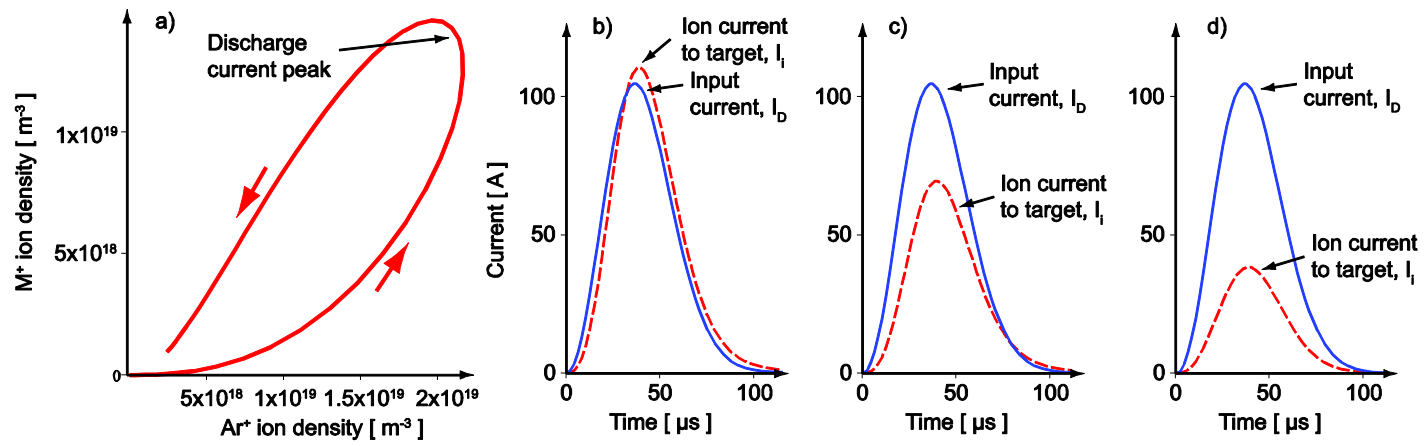

Figure 3. Results from the IRM I. a) Density plot of $n_{M^{+}}$versus $n_{A r^{+}}$for the parameters $\beta_{A r^{+}}=0.88, \gamma_{s e}=0.1$. The plots $\mathbf{b}-\mathbf{d}$ show modeled ion current to target compared to measured discharge current for the parameters b) $\beta_{A r^{+}}=0.88, \gamma_{s e}=0.1$ c) $\beta_{A r^{+}}=0.88, \gamma_{s e}=0.06$ and d) $\beta_{A r^{+}}=0.36, \gamma_{s e}=0.06$.

determined by the relative ion diffusion speeds to, and sizes of, the different boundaries to the ionization region. Here, two cases has been studied, assuming the diffusive ion flow to the target (at the sheath edge) to be at the ion acoustic speed using the electron temperature calculated in the model (2.7 eV at current maximum) for both cases. The speed at the boundary to the bulk plasma is taken as one of two extremes: the thermal speed at $300 \mathrm{~K}$ filling gas temperature (corresponding to distant substrate and walls, and probably most resembling the chosen reference discharge), or the ion acoustic speed (corresponding to a hypothetical case with close-lying substrate and walls). The model assumes a $2 \mathrm{~cm}$ thick and $5 \mathrm{~cm}$ wide ionization region centered over the race track giving approximate values $\beta_{A r^{+}} \approx 0.88$ (for distant chamber walls) and $\beta_{A r^{+}} \approx 0.36$ (for close-lying chamber walls). Figure 3 shows results from three IRM I runs with different $\gamma_{s e}$ and $\beta_{A r^{+}}$. The first run (Figure $3 \mathrm{a}$ and $3 \mathrm{~b}$ ) has parameters $\gamma_{s e}=0.1$ and $\beta_{A r^{+}} \approx 0.88$. Figure $3 \mathrm{~b}$ shows the calculated ion current to the target $I_{i}$ 
and the input current $I_{D}(t)$. A discharge with these $\gamma_{s e}$ and $\beta_{A r^{+}}$seems to be possible, since $I_{i}$ is quite close to $I_{D}$. This is also consistent with Eq. (1) which, with these $\gamma_{s e}$ and $\beta_{A r^{+}}$and at current maximum $\left(U_{D}=430 \mathrm{~V}\right)$, gives $\Pi_{\text {Townsend }}=1.1$. However, a look at the $\mathrm{Ar}^{+}$and $\mathrm{M}^{+}$densities (Figure 3a) shows that during the pulse there is an approximate average $60 / 40 \mathrm{Ar}^{+} / \mathrm{M}^{+}$ratio. For $\mathrm{M}^{+}, \gamma_{s e} \approx 0$ and the assumed constant $\gamma_{s e}$ $=0.1$ during the pulse is therefore unrealistic. Closer to reality is the run in Figure 3c, where $\gamma_{s e}=0.06$. This discharge can probably not be sustained since $I_{i}<I_{D}$. Finally, Figure $3 \mathrm{~d}$ shows the effect of $\gamma_{s e}=0.06$ combined with enhanced ion losses to closelying substrate and walls $\left(\beta_{A r^{+}} \approx 0.36\right)$. Under these conditions a discharge with $E_{z}=0$ is clearly not possible.

These results from the IRM I are in good agreement with those obtained from Eq. (1), using the same $\gamma_{s e}, \beta_{A r^{+}}$, and taking $U_{D}$ at current maximum. This indicates that the simple model in Figure 2 captures the key elements of the much more complicated discharge physics. Let us therefore extend the Townsend product to make a crude estimate of how large $E_{z, \text { ioniz }}$ fields would be needed in the ionization region to sustain the $A r^{+}$ion particle balance. With $E_{z \text {,ioniz }} \neq 0$, more ions are redirected towards the target and the value of $\beta_{A r^{+}}$increases, with $\beta_{A r^{+}}=1$ as a definite upper limit. Furthermore, there will be increased ionization because the bulk electrons are heated directly. Assuming that in the ionization region on the average $50 \%$ of the current is carried by electrons, the total power input to the electrons is $P_{e}=I_{D} \gamma_{s e}\left(U_{D}-U_{I R}\right)+0.5 I_{D} U_{I R}$. Taking into account the different costs of ionization of the secondary electrons (hot) and the bulk electrons (cold), the Townsend product becomes 


$$
\Pi_{\text {Townsend }}=\left(\gamma_{s e} \frac{U_{D}-U_{I R}}{U_{i, E F F, h}}+0.5 \frac{U_{I R}}{U_{i, E F F, c}}\right) \beta_{A r^{+}} .
$$

When $U_{I R}=0$, the earlier Eq. (1) is recovered. For a numerical example we take current maximum $\left(U_{D}=430 \mathrm{~V}\right), \Pi_{\text {Townsend }}=1$ for steady-state, $\beta_{A r^{+}}$and $\gamma_{s e}$ as in Figure 3c, $U_{i, E F F, c}=60 \mathrm{eV}$ and $U_{i, E F F, h}=35$ (see footnote *). This inserted in Eq. (2) gives $U_{\mathrm{IR}} \approx 60 \mathrm{~V}$, in the higher end of the observed range $7-60 \mathrm{~V}$. The absolute value of this estimate must be regarded as very uncertain, but it clearly indicates that $E_{z, \text { ioniz }}$ fields are likely for normal operating HiPIMS conditions.

\section{Transport driven electric fields $E_{z, \text { trans }}$}

In the bulk plasma, electrons have to move across the magnetic field lines in order to arrive at the anode and close the current loop, as illustrated in Figure 1c. In this region transport driven fields $E_{z, \text { trans }}$ will appear in order to make that possible. Cross-B discharge current density and electric fields are related through the component along $E_{\perp}$ of the generalized Ohm's law [14,15]

$$
E_{\perp}=\frac{J_{\perp}}{\sigma_{P}}=J_{\perp} /\left(\frac{n_{e} e}{B} \cdot \frac{\omega_{g e} \tau_{E F F}}{1+\left(\omega_{g e} \tau_{E F F}\right)^{2}}\right)
$$

Eq. 3 can be used to model the potential $V(r, z)$, and the current density distribution $\mathbf{J}(r, z)$, provided that $I_{D}, n_{e}$ (electron density), $\omega_{g e} \tau_{E F F}$ (electron angular gyration frequency times the effective collision time), and $\mathbf{B}(r, z)$ are known $[16]^{\dagger} . \sigma_{P}$ is the Pedersen conductivity across $\mathbf{B}(r, z)$.

Let us look at what is known regarding the needed input data $n_{e}$ and $\omega_{g e} \tau_{E F F}$. Optical emission measurements by Hala et al. [17] show that the bulk plasma can

\footnotetext{
${ }^{\dagger}$ In reference 16, it is also assumed that $\mathbf{E} \cdot \mathbf{B}=0$.
} 
expand with a speed in the range $1-3.5 \mathrm{~km} / \mathrm{s}$ depending on species and pressure. From Langmuir and magnetic probe measurements $[10,11]$, the expansion speed in our
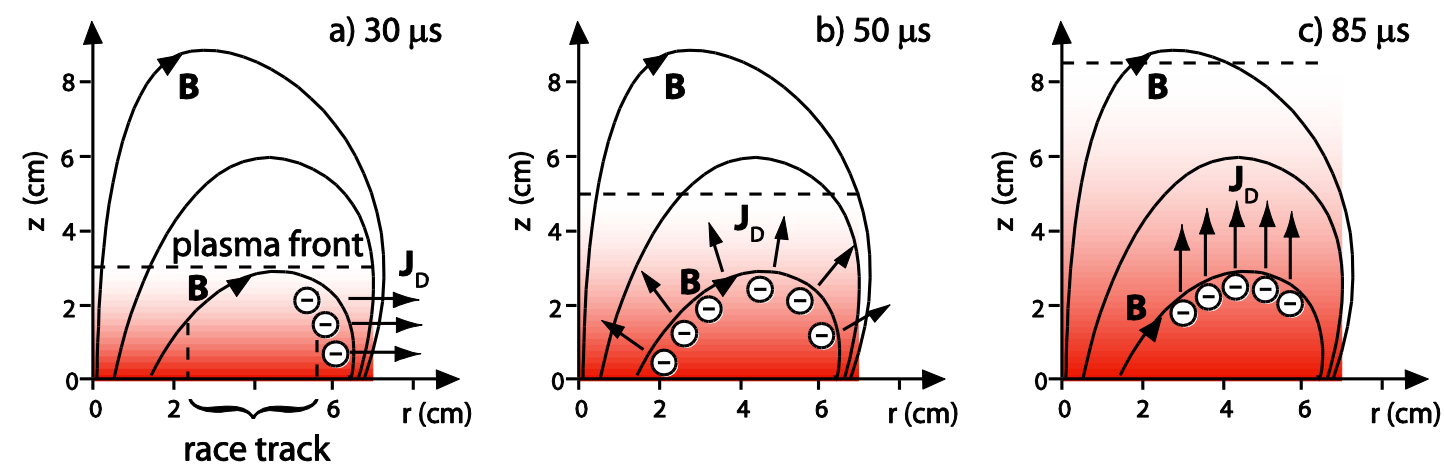

Figure 4. Electron drift directions across B in the HiPIMS discharge at different times: a) $30 \mu \mathrm{s}$

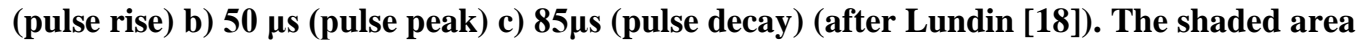
schematically illustrates the expansion of plasma with a front speed of $1 \mathrm{~km} / \mathrm{s}[10,11]$. The dashed line represents the plasma front.

reference magnetron is estimated to be about $1 \mathrm{~km} / \mathrm{s}$. Recent measurements of the internal currents [18] have shown how these evolve in space and time. In Figure 4, the temporal change of the cross-B electron transport during a HiPIMS pulse is proposed based on these measurements. During the initial part of the pulse, when there is little plasma in the bulk volume, most of the cross-B current flows radially close to the target (Figure 4a). As the plasma expands into the bulk volume the current system gradually changes, on a $50-100 \mu$ s time scale, towards a vertical current channel (figure 4c) resembling that in direct current magnetron sputtering (DCMS).

Now let us turn to $\omega_{g e} \tau_{E F F}$. In the bulk plasma the current is carried mainly by electrons, and the relation $\omega_{g e} \tau_{E F F} \approx J_{\varphi} / J_{\perp}[13,19]$ can be used to obtain $\omega_{g e} \tau_{E F F}$ from $J_{\varphi} / J_{\perp}$ measurements. This has earlier been done in DCMS [20], in pulsed DCMS [21], and in HiPIMS [10,12], but always built on some assumed distribution of $J_{\perp}$. In view of the now known temporal redistribution shown in Figure 4 they must therefore be regarded as uncertain. Still, the trend is clear. In DCMS, $\omega_{g e} \tau_{E F F}$ lies 
within a factor of two from the Bohm value [14] $\omega_{g e} \tau_{E F F}=16$, a result also reported from recent particle in cell Monte Carlo simulations [22]. In pulsed DCMS and HiPIMS the values are significantly lower, in the studies above uniformly reported as

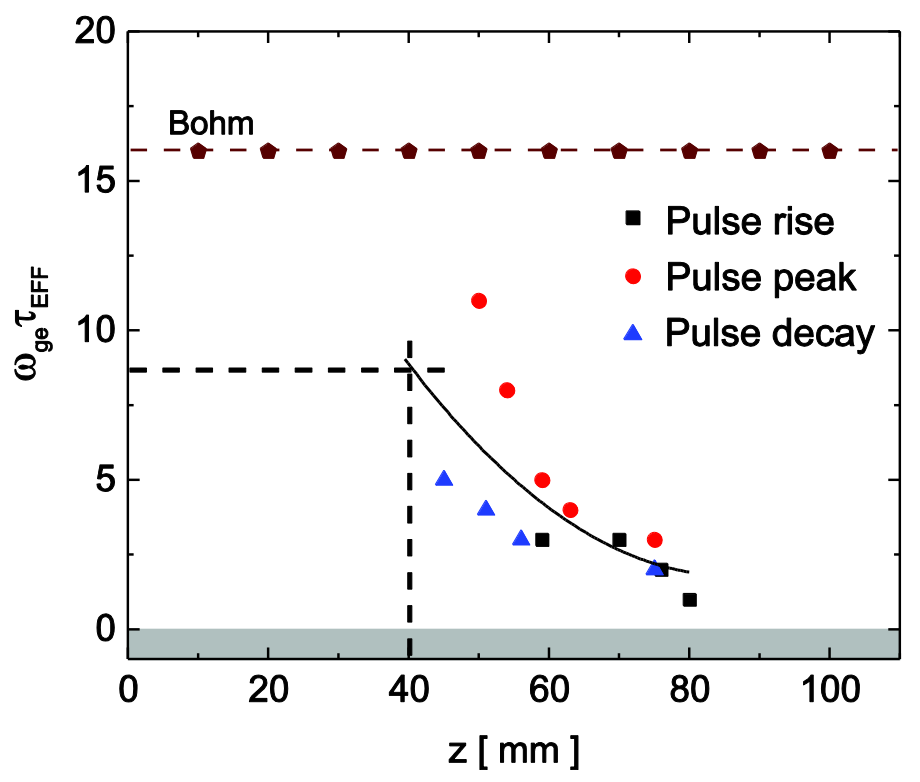

Figure 5. Measured $\omega_{g e} \tau_{E F F}$ above the target race track for three different times during the HiPIMS discharge (after Lundin [18]).

$\omega_{g e} \tau_{E F F} \approx 2$. This now has to be revised. The first space- and time resolved current measurements [18] shows a more complicated picture, see Figure 5, with higher $\omega_{g e} \tau_{E F F}$ values closer to the target, and also with some time variation. We here only note that around $z=4 \mathrm{~cm}$ a "best estimate" could be $\omega_{g e} \tau_{E F F} \approx 8$, but with an uncertainty by perhaps a factor of two.

In view of the uncertainties in both $n_{e}$ and $\omega_{g e} \tau_{E F F}$, quantitative modeling of transport driven $E_{z, \text { trans }}$ based on Eq. (3) is probably not feasible at the moment. It is here only demonstrated that such fields are likely, and how sensitive they are to the uncertainty in $\omega_{g e} \tau_{E F F}$. Figure 6 shows results from the bulk plasma model of Brenning et al. [16]. Input parameters are the magnetic field $\mathbf{B}(r, z)$ of our reference 
magnetron, the peak current $I_{D}=105 \mathrm{~A}$, and density estimates based on earlier probe measurements $[10,11]$ in the same device. $\omega_{g e} \tau_{E F F}$ is assumed to be constant in the volume. As can be seen in Figure 6, the earlier proposed value $\omega_{g e} \tau_{E F F}=2$ (which

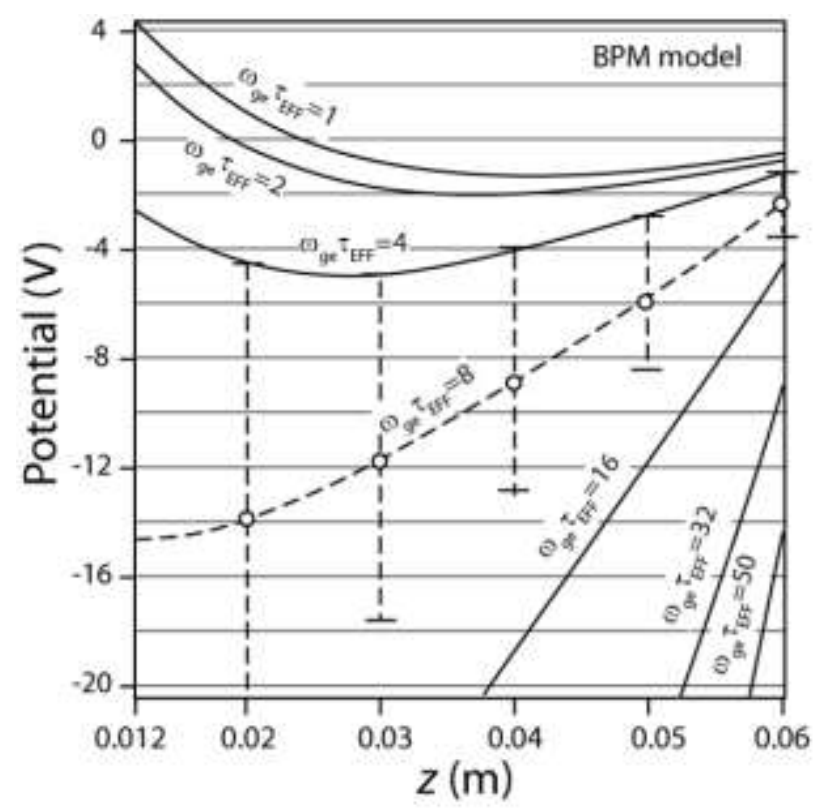

Figure 6. Calculated potential profiles above the target race track for different $\omega_{g e} \tau_{E F F}$ using the bulk plasma model. The dashed curve denotes $\omega_{g e} \tau_{E F F}=8$ and the dashed bars denote a range of \pm 4 around that value. The results are adapted from Brenning et al. [16]

was believed to be correct at the time of the publication [16]), results in unrealistically low $E_{z}$, with potential variations of only a few volts above the race track. The Bohm value $\omega_{g e} \tau_{E F F} \approx 16$ would give $\sim 50 \mathrm{~V}$ from sheath edge to bulk plasma, while the "best estimate" $\omega_{g e} \tau_{E F F}=8$ from Figure 5 results in $\sim 10 \mathrm{~V}$. This is large enough to significantly increase the $\mathrm{M}^{+}$back-attraction and also consistent (within a factor of two) with probe measurements in the same device [5]. 


\section{Summary and discussion}

Recent measurements in two different HiPIMS devices $[5,6]$ have shown electric fields $E_{z}$ in the plasma that are strong enough to cause considerable back-attraction of the ionized sputtered species $M^{+}$. The associated potential uphill extended a distance of $5-6 \mathrm{~cm}$ from the sheath edge into the plasma, and had amplitudes in the range $6-$ $70 \mathrm{~V}$. HiPIMS discharges should be possible to optimize with respect to such electric fields since they in these experiments varied with parameters that can be externally controlled as the magnetic field strength [6], the gas pressure [5,6], and the applied power [6]. It is also very likely that they can be influenced by varying the pulse shape and length: the $E_{z}$ fields are generally stronger closer to the target, and during the phases of breakdown and current rise [6].

Outside the sheath, on a larger scale length, there is a always a presheath [14] in which the ions are accelerated to the Bohm speed by a potential drop $\Phi \geq k_{B} T_{e} /(2 e)$, in HiPIMS magnetrons at most a few volts. Let us call the associated field $E_{z \text {,presheath }}$. We here propose that in addition to $E_{z \text {,presheath }}, E_{z}$ fields in the plasma are driven mainly by two mechanisms, and that these dominate in different regions of the discharge. Ionization driven fields $E_{z, \text { ioniz }}$ can be expected in an ionization region extending a few $\mathrm{cm}$ from the target, approximately corresponding to the negative glow in dc glow discharges. These $E_{z, \text { ioniz }}$ fields are forced by the energy and particle balance conditions for a self sustained discharge. The other type of fields are transport driven $E_{z, \text { trans }}$, needed to carry the electrons across the magnetic field lines in the surrounding bulk plasma. The limit between these two mechanisms should not be expected to be sharp: there is a need to carry electrons across the magnetic field also in the ionization region, and there is ionization also in the bulk plasma. Where 
different mechanisms overlap, the strongest required field should result, i.e. $E_{z}=\max \left(E_{z, \text { presheath }}, E_{z, \text { ioniz }}, E_{z, \text { trans }}\right)$

Modeling of these two types of $E_{z}$ fields (see sections 2 and 3 above) indicates that both are likely to arise under normal HiPIMS operating conditions, and also that they are of the same order as those observed. Identified risk factors, leading to high $E_{z}$ in the models, are: (1) close-lying substrates and walls that result in large ion loss fluxes, and a high wall loss rate of electron energy, (2) fast rising currents with the need of an ionization rate per electron that is far above that in steady-state, (3) gas depletion caused by long and/or high energy pulses, which forces the discharge towards the self-sputtering mode in which the low $\gamma_{s e}$ for $M^{+}$ions is an additional problem, (4) a low density in the bulk plasma which forces electron transport to go across the stronger magnetic fields closer to the target, and (5) strong magnetic fields above the race track which gives higher transport driven $E_{z, \text { trans }}$ for a given current density.

Even with a known potential profile $V(r, z, t)$, the effective back-attraction is a complicated function and depends also the plasma profile $n_{e}(r, z, t)$. Out of the sputtered energy distribution of neutrals emitted from the target, $f\left(E_{\text {sputtered }}\right)$, the components with higher velocity $v_{z}$ will on the average become ionized at larger distances from the target, have a larger directed energy $m_{M} v_{z}^{2} / 2$, and also a lower remaining potential hill to climb. The combination of these effects leads to a highenergy filtering of the $\mathrm{M}^{+}$flux that can reach the substrate. A proper evaluation of the back-attraction would require at least Monte Carlo simulations over $f\left(E_{\text {sputtered }}\right)$ including collisions with the background gas. 


\section{Acknowledgements}

This work was partly supported by the Swedish Research Council, the Swedish

Foundation for Strategic Research, the European Collaboration in Science and Technology (COST Action: MP0804), the ANH HiPPoPP (French Government Research Agency), and the Romanian ministry of Education, Research, Youth and Sport, Grant IDEI 540/2009.

\section{References}

[1] Helmersson U, Lattemann M, Bohlmark J, Ehiasarian A P and Gudmundsson J T 2006 Thin Solid Films 5131

[2] Bohlmark J, Lattemann M, Gudmundsson J T, Ehiasarian A P, Aranda Gonzalvo

Y, Brenning N and Helmersson U 2006 Thin Solid Films 5151522

[3] Christie D J 2005 J. Vac. Sci. Technol. A 23330

[4] Andersson J and Anders A 2009 Phys. Rev. Lett. 102045003

[5] Sigurjonsson P 2008 Master's Thesis: Spatial and temporal variation of the plasma parameters in a high power impulse magnetron sputtering (HiPIMS) discharge (Reykjavik: Faculty of Engineering, University of Iceland)

[6] Mishra A, Kelly P J and Bradley J W 2010 Plasma Sources Sci. Technol. 19 045014

[7] Lieberman M A and Lichtenberg A J 1994 Principles of Plasma Discharges and Materials Processing (New York: John Wiley \& Sons) p 81

[8] Samuelsson M, Lundin D, Jensen J, Raadu M A, Gudmundsson J T and Helmersson U 2010 Surf. Coat. Technol. 205591 
[9] Raadu M A, Axnäs I, Gudmundsson J T and Brenning N 2010 manuscript in preparation

[10] Bohlmark J, Helmersson U, VanZeeland M, Axnäs I, Alami J and Brenning N 2004 Plasma Sources Sci. Technol. 13654

[11] Bohlmark J, Gudmundsson J T, Alami J, Lattemann M and Helmersson U 2005 IEEE Trans. Plasma Sci. 33346

[12] Lundin D, Helmersson U, Kirkpatrick S, Rohde S and Brenning N 2008 Plasma Sources Sci. Technol. 17025007

[13] Lundin D, Larsson P, Wallin E, Lattemann M, Brenning N and Helmersson U 2008 Plasma Sources Sci. Technol. 17035021

[14] Chen F F 1984 Introduction to Plasma Physics and Controlled Fusion Vol. I: Plasma Physics (New York: Plenum)

[15] Spitzer L 1962 Physics of Fully Ionized Gases $2^{\text {nd }} e d$. (New York: John Wiley \& Sons)

[16] Brenning N, Axnäs I, Raadu M A, Lundin D and Helmerson U 2008 Plasma Sources Sci. Technol. 17045009

[17] Hala M, Viau N, Zabeida O, Klemberg-Sapieha J W and Martinu L 2010 J. Appl. Phys. 107043305

[18] Lundin D 2010 Doctoral Thesis: The HiPIMS Process, (Linköping: Linköping University)

[19] Rossnagel S M and Kaufman H R 1987 J. Vac. Sci. Technol. A 52276

[20] Bradley J W, Thompson S and Aranda Gonzalvo Y 2001 Plasma Sources Sci.

Technol. 10490

[21] Vetushka A and Bradley J W 2007 J. Phys. D: Appl. Phys. 402037 
[22] E. Bultinck E, Mahieu S, Depla D and Bogaerts A 2010 J. Phys. D: Appl. Phys. 43292001 